\title{
When the arteries get tough, the tougher do not get going
}

\author{
Charalambos Vlachopoulos, Dimitrios Terentes-Printzios and Christodoulos Stefanadis
}

Hypertension Research (2011) 34, 793-794; doi:10.1038/hr.2011.49; published online 28 April 2011

$A$ ortic stiffness increases significantly and nonlinearly with age. ${ }^{1}$ Carotid-femoral pulse wave velocity (PWV), the gold-standard index for aortic stiffness, increases monotonically from young adulthood throughout the entire human lifespan (Figure 1). ${ }^{2}$ The age trajectory of carotid-femoral $\mathrm{PWV}$ is concave, with the slope increasing modestly at midlife and then sharply after 50 years of age. Augmentation index (AIx), a measure of wave reflections that is related to arterial stiffness, increases in parallel with PWV through midlife, but then reaches a plateau or falls slightly after 60 years of age (Figure 1). ${ }^{2}$ In younger individuals, AIx rises steeply with age, whereas PWV does not. Conversely, in older individuals, AIx changes little, but aortic PWV increases steeply. $^{2}$ These suggest that, AIx and PWV, although related, may provide different but potentially complementary information. Specifically, AIx might be a more sensitive marker of arterial aging in younger individuals, whereas aortic PWV might be a more sensitive marker in older individuals. Therefore, to fully assess the impact of aging and risk factors on large artery hemodynamics, both markers might need to be assessed.

Prevention of cardiovascular disease is greatly aided by the identification of proper biomarkers. Among the recently proposed novel biomarkers of cardiovascular disease risk, aortic stiffness and wave reflections indices stand out as important risk predictors for future events. Carotid-femoral PWV is a robust predictor of cardiovascular risk and all-cause mortality. We recently meta-analyzed aortic PWV data from 17 available

Dr C Vlachopoulos is at the Peripheral Vessels Unit, 1st Department of Cardiology, Hippokration Hospital, Athens Medical School, Profiti Elia 24, Athens 14575, Greece.

E-mail: cvlachop@otenet.gr published studies for 15877 subjects followed up for a mean of 7.7 years. ${ }^{3}$ Several populations such as patients with hypertension, diabetes, end-stage renal disease and subjects from the general population were included. Nevertheless, among these studies only three investigated the predictive ability of PWV in the elderly. ${ }^{4-6}$ With regard to indices of

central hemodynamics, accumulating data support their ability to predict CV events and mortality. In a subsequent meta-analysis, we demonstrated that a $10 \%$ increase of central AIx is associated with a relative risk of 1.29 for cardiovascular events and of 1.38 for all-cause mortality. ${ }^{7}$ However, in the elderly such data are lacking.
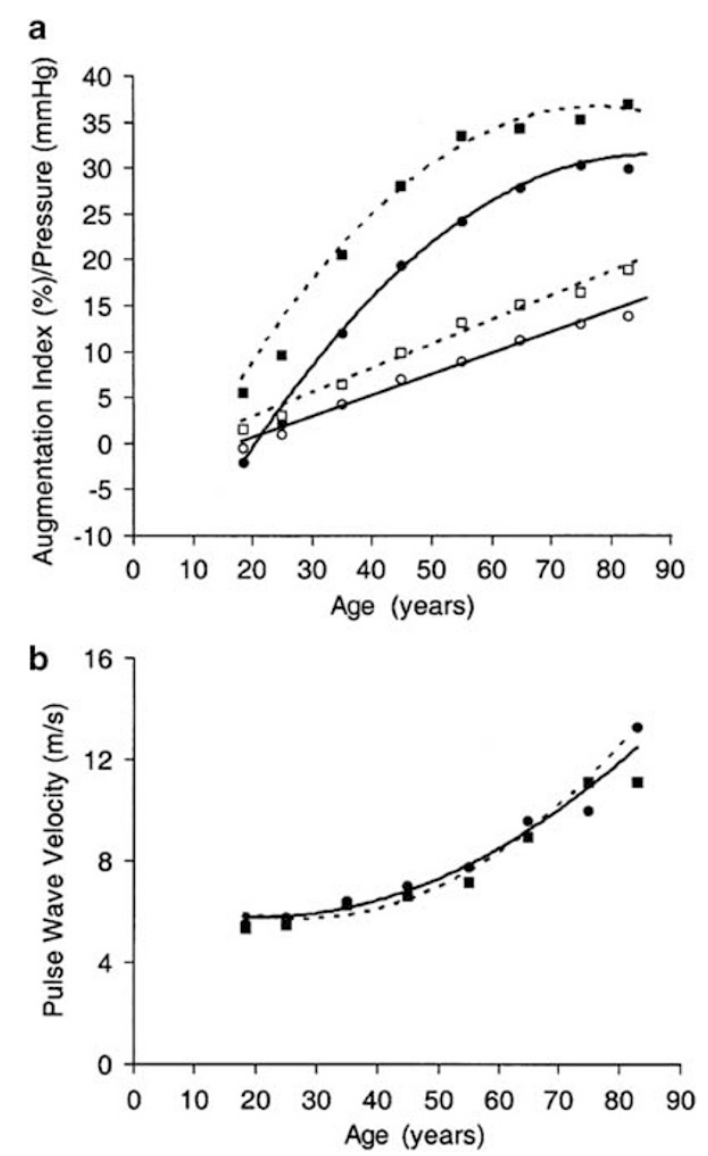

Figure 1 Regression curves representing the effect of age on aortic stiffness and wave reflection indices for males (circles, solid lines) and females (squares, dashed lines). Panel a represents augmentation pressure (open circles/open squares) and augmentation index (closed circles/closed squares). Panel b represents aortic pulse wave velocity (from McEniery et al. ${ }^{2}$ ). 


\section{All-cause mortality}

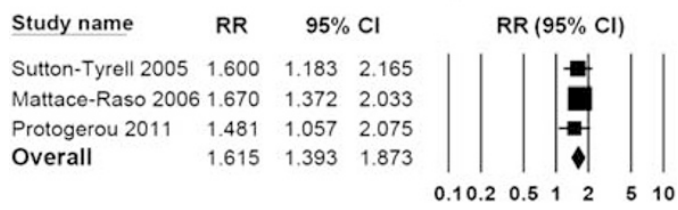

Figure 2 Relative risk (RR) and 95\% confidence interval $(\mathrm{Cl})$ for high aortic pulse wave velocity and all-cause mortality. Studies are listed chronologically. Boxes represent the RR and lines represent the $95 \% \mathrm{Cl}$ for individual studies. The diamond and its width represent the pooled RR and the $95 \% \mathrm{Cl}$, respectively.

Aiming at filling the knowledge gap in this fragile age group, the study of Protogerou et al., ${ }^{8}$ provides important data. The PROTEGER study demonstrates that in elderly patients with cardiovascular disease there is a synergistic effect on survival when both elevated aortic stiffness and pressure wave reflections are present, supporting the notion that aortic stiffness and wave reflection indices impart complementary information.

A finding of the PROTEGER study that merits further scrutiny is that neither PWV nor AIx were predictive of all-cause mortality when these two parameters were evaluated alone. Although larger populations and a longer follow-up period are desirable, this finding can be interpreted in light of the following: In our aforementioned meta-analysis on aortic PWV, there was a lesser predictive ability of stiffness for clinical events in older end-stage renal disease patients. ${ }^{3}$ Plausible explanations may include a 'selection' phenomenon, with survivors who reach an older age being less vulnerable to the harmful effects of arterial stiffening. Furthermore, at the occasion of this commentary, we performed a sub-analysis of data in elderly subjects, by including the study of Protogerou et al., ${ }^{8}$ and two of the three abovementioned studies on elderly subjects that provided the relevant data. ${ }^{4,5}$ The pooled relative risks of all-cause mortality were higher for high-aortic PWV compared with low-stiffness elderly subjects (relative risk: 1.615, 95\% confidence interval: 1.393-1.873, $P<0.001$ ), corresponding to a risk increase of $61.5 \%$ (Figure 2). This value, although significant, is lower compared with the risk increase of $90 \%$, regarding the overall population. ${ }^{3}$ Taken together, these pieces of evidence supports the notion that increased PWV is less predictive in the elderly population.

Although aortic stiffness and wave reflection indices are traditionally conceived as predictors of cardiovascular risk, they are powerful predictors of all-cause mortality as well. ${ }^{3}$ On this wavelength, Protogerou et al. ${ }^{8}$ insightfully focus on all-cause mortality as the main outcome of interest. Although by virtue of their pathophysiological basis, aortic stiffness and wave reflections indices are expected to predict cardiovascular events, their association with all-cause mortality lacks a prima facie explanation. Although pathophysiological explanations are not readily identifiable, their ability to predict events could reflect the existence of common pathogenetic mechanisms, such as aging, inflammation and oxidative stress, over a wide range of conditions.

When the arteries get tough, the tougher do not get going, as PWV remains a powerful biomarker, despite losing some of its predictive ability at older age. Most importantly, a holistic approach for evaluating arterial function, in which complementary arterial biomarkers, such as PWV and wave reflection indices, are combined, ${ }^{8-11}$ has the potential to increase our ability to predict the risk of both cardiovascular events and all-cause mortality.
1 Reference Values for Arterial Stiffness' Collaboration. Determinants of pulse wave velocity in healthy people and in the presence of cardiovascular risk factors: 'establishing normal and reference values'. Eur Heart J 2010; 31: 2338-2350.

2 McEniery CM, Yasmin, Hall IR, Qasem A, Wilkinson IB, Cockcroft JR, ACCT Investigators. Normal vascular aging: differential effects on wave reflection and aortic pulse wave velocity: the Anglo-Cardiff Collaborative Trial (ACCT). J Am Coll Cardiol 2005; 46: 1753-1760.

3 Vlachopoulos C, Aznaouridis K, Stefanadis C. Prediction of cardiovascular events and all-cause mortality with arterial stiffness: a systematic review and metaanalysis. J Am Coll Cardiol 2010; 55: 1318-1327.

4 Meaume S, Benetos A, Henry OF, Rudnichi A, Safar $\mathrm{ME}$. Aortic pulse wave velocity predicts cardiovascular mortality in subjects $>70$ years of age. Arterioscler Thromb Vasc Biol 2001; 21: 2046-2050.

5 Sutton-Tyrrell K, Najjar SS, Boudreau RM, VenkitachaIam L, Kupelian V, Simonsick EM, Havlik R, Lakatta EG, Spurgeon H, Kritchevsky S, Pahor M, Bauer D, Newman A, Health ABC Study. Elevated aortic pulse wave velocity, a marker of arterial stiffness, predicts cardiovascular events in well-functioning older adults. Circulation 2005; 111: 3384-3390.

6 Mattace-Raso FU, van der Cammen TJ, Hofman A, van Popele NM, Bos ML, Schalekamp MA, Asmar R, Reneman RS, Hoeks AP, Breteler MM, Witteman JC. Arterial stiffness and risk of coronary heart disease and stroke. The Rotterdam Study. Circulation 2006; 113: 657-663.

7 Vlachopoulos C, Aznaouridis K, O'Rourke MF, Safar ME, Baou K, Stefanadis C. Prediction of cardiovascular events and all-cause mortality with central haemodynamics: a systematic review and meta-analysis. Eur Heart J 2010; 31: 1865-1871.

8 Protogerou AD, Safar ME, Papaioannou TG, Zhang Y, Agnoletti D, Papadogiannis D, Blacher J. The combined effect of aortic stiffness and pressure wave reflections on mortality in the very old with cardiovascular disease: the PROTEGER Study. Hypertens Res 2011; 34: 803-808.

9 Vlachopoulos C, Terentes-Printzios D, Stefanadis C. Arterial stiffness and carotid intima-media thickness: together they stand. Hypertens Res 2010; 33: 291-292.

10 Tzortzis S, Ikonomidis I, Lekakis J, Papadopoulos C, Triantafyllidi H, Parissis J, Trivilou P, Paraskevaidis I, Anastasiou-Nana M, Kremastinos DT. Incremental predictive value of carotid intima-media thickness to arterial stiffness for impaired coronary flow reserve in untreated hypertensives. Hypertens Res 2010; 33: 367-373.

11 Stamatelopoulos KS, Kalpakos D, Protogerou AD, Papamichael CM, Ikonomidis I, Tsitsirikos M, Revela I, Papaioannou TG, Lekakis JP. The combined effect of augmentation index and carotid intima-media thickness on cardiovascular risk in young and middle-aged men without cardiovascular disease. $\mathrm{J}$ Hum Hypertens 2006; 20: 273-279. 